\title{
DOCEAMUS
}

doceamus...let us teach

\section{How Content Matters}

\section{Alain Schremmer}

Nowadays, about half of the college student population is in two-year colleges, and as they enter, the overwhelming majority of these students are totally unprepared for whatever college-level courses they have to take. Unfortunately, the belief that all that "unprepared students" need is to learn "mathematical facts" and acquire computational skills, together with the concomitant belief that, to that end, better pedagogy is all that is needed has caused a complete atomization of the contents and thus complete reliance on memorization. Also, it is unrealistic to expect that even two semesters of Developmental Mathematics can remedy so many lost years. It is thus rather unsurprising that during the near forty years of its existence, Developmental Mathematics has never worked. ${ }^{1}$

The premise for what is being proposed here is, as physicist Hestenes, of Geometric Algebra fame, wrote in his Oersted Medal lecture, that "[That] course content is taken [by many] as given [...] ignores the possibility of improving pedagogy by reconstructing course contents." The aim is to call for

Alain Schremmer is associate professor of mathematics at the Community College of Philadelphia. His email address is aschremmer@ccp.edu.

Members of the Editorial Board for Doceamus are: David Bressoud, Roger Howe, Karen King, William McCallum, and Mark Saul.

${ }^{1}$ At least when measured in terms of subsequent success instead of throughput. For instance, at my institution less than one quarter of one percent (0.24\%) of the students starting on the route Arithmetic-Basic Algebra-Intermediate Algebra-Precalculus I-Precalculus II-Calculus I ever complete Calculus I.

DOI: http://dx.doi.org/10.1090/noti961 mathematicians to engage in such reconstructions and to show that it can be interesting.

What follows is just one-very sparse-example of how a reconstruction of the contents of Developmental Arithmetic and Algebra, together with a corresponding reconstruction of the contents of Precalculus and Differential Calculus, could lead to a three-semester, 5-4-4 sequence realistically accessible to developmental students. In total opposition to what is to be found in all currently available commercial texts, the idea is merely to use systematically a few powerful concepts with one thing flowing smoothly into the next.

\section{Arithmetic}

We start with the construction of a symbolic system for representing on paper collections of real-world items. We use number phrases such as 3 apples, consisting of a numerator to represent the number of items and a denominator to represent the kind of items. The point here is twofold: (i) This is something completely self-contained that can be dealt with to the complete satisfaction of the students, and (ii) this sets the stage for future distinctions such as between $\frac{3}{7}+\frac{2}{7}$, which is equal to $\frac{5}{7}$; and $\frac{3}{5}+\frac{2}{7}$, which is a (linear) combination (aka vector); ${ }^{2}$ and as between $3 x^{7}+2 x^{7}$, which is equal to $5 x^{7}$; and $3 x^{5}+2 x^{7}$, which is a combination.

In fact, we immediately use combinations to construct the Decimal Numeral System: 42.75 Dollars is introduced as shorthand for the combination 4 DEKADollars + 2 Dollars + 7 DECIDollars + 5 CENTIDollars.

\footnotetext{
$2+$ being read here as "and".
} 
A further stage is to discuss operations which we see the old way, that is, as unary. Here again, this has two purposes: (i) to stick to the real world, where, for instance, we attach a collection to a collection, and (ii) to turn the concept of unary operation into that of a function.

The discussion of reverse problems leads naturally to the introduction of signed numbers in the case of addition and to decimal numbers in the case of multiplication. We thus arrive at $\mathbb{D}$, the set of signed decimal numbers.

However, if multiplication is introduced as dilation, we also look at it as "co-multiplication", as in -3 apples $\odot-5 \frac{\text { cents }}{\text { appte }}$, which, given that nobody has ever objected to the fact that getting rid of bad apples is a plus, immediately gives +15 cents.

We introduce fractions as code for division as in $\frac{1}{3}=0+[\ldots]=0.3+[\ldots]=0.33+[\ldots]$, where [...] is read "something too small to matter here". ${ }^{3}$ Eventually, quarter is defined as "of which it takes 4 to exchange for 1 dollar", from which the rest follows naturally, e.g., $\frac{7}{4}$ dollar is equivalent to the combination 1 dollar +3 quarters. Then, if dimes can be exchanged at the rate of $2 \frac{\text { nickels }}{\text { dime }}$ and quarters at $5 \frac{\text { nickels }}{\text { quarter }}$, then 3 dimes -2 quarters can be exchanged for

$$
\begin{gathered}
\text { (+3 dimes,-2 quarters)@ }\left[\begin{array}{c}
+2 \frac{\text { nickels }}{\text { dime }} \\
+5 \frac{\text { nickels }}{\text { quarter }}
\end{array}\right] \\
=-4 \text { nickels, }
\end{gathered}
$$

with nickels in the underlying field. In other words, we get $\frac{3}{10}-\frac{2}{4}=-\frac{4}{20}$.

\section{Algebra}

We now deal with the mechanics of solving reverse problems. After the relation symbols $=, \neq,>,<, \geqq$, have been introduced, we introduce "(in)equations" as open sentences sifting a solution subset out of a data set. We discuss the differences between the case of data sets consisting of whole numbers and that of data sets consisting of decimal numbers. Hence to specify a solution subset, we use the notion of an inequation problem, i.e., of a data set together with an inequation. We then spend a considerable amount of time dealing successively, mostly in the case of decimal data sets, with basic problems (e.g., $x \leqq-352.94)$, translation problems (e.g., $x \oplus-37.183>-352.94$ ), dilation problems (e.g., $x \odot-23.1327 \neq-822.08$ ), and affine problems, using exclusively the standard approach: (i) Find the boundary points by solving the associate equation. (ii) Test the boundary points against the given inequation. (iii) In each one of the intervals determined by the boundary points, test a point

\footnotetext{
${ }^{3}[\ldots]$ can thus be seen as an undifferentiated precursor of Landau's $o\left[h^{n}\right]$.
}

against the inequation and use the Pasch Theorem, which says that "whatever is true of the test point is true of all points in the interval." We then spend time with double problems, i.e., problems involving two inequations bound by one of three connectors: BOTH, EITHER ONE OR BOTH, EITHER ONE BUT NOT вотн. We start with double basic problems and work our way up to double affine problems. ${ }^{4}$

Finally, with an unfortunate hiatus, we move to the notion of power with monomial functions; i.e., instead of multiplying the coefficient $a$ by a single copy of the input $x$, as with dilation functions, we either

- multiply $a$ by $n$ copies of the input $x$ :

$$
x \underset{n \text { copies of } x}{\stackrel{a \text { MONOMIAL }_{+n}}{\longrightarrow} a \text { MONOMIAL }_{+n}(x)} \text { aka } a x^{+n}
$$

or

- divide $a$ by $n$ copies of the input $x$ :

$$
\begin{gathered}
x \stackrel{a \text { MONOMIAL }_{-n}}{\longrightarrow} a \text { MONOMIAL }_{-n}(x) \\
=\underbrace{\frac{a}{x \cdot x \cdots x \cdot x}}_{n \text { copies of } x} \text { aka } a x^{-n} .
\end{gathered}
$$

In other words, $x^{ \pm n}$ is just code to be read as "multiplied (resp. divided) by $n$ copies of $x$ " and generalizes the code $10^{n}$ that we used with decimal numbers and initially read as " 1 followed/preceded by $n$ zeros".

Computing with monomials, we find that:

1. From the multiplicative viewpoint, the code lets us write $3 x^{+2} \cdot 4 x^{+3}=3 \cdot x \cdot x \cdot 4 \cdot x \cdot x$. $x=3 \cdot 4 \cdot x \cdot x \cdot x \cdot x \cdot x=12 x^{+5}$. Similarly, we have $3 x^{+2} \cdot 4 x^{-3}=3 \cdot x \cdot x \cdot \frac{4}{x \cdot x \cdot x}=3 \cdot 4 \cdot \frac{x \cdot x}{x \cdot x \cdot x}=$ $12 x^{-1}$. Eventually we discover that "o-plussing (resp. o-minussing) the exponents" does the job automatically for the multiplication (resp. division) of monomials.

2. From the additive viewpoint, $x^{ \pm n}$ behaves like a denominator, e.g., $5 x^{-4}+2 x^{-4}=7 x^{-4}$, but $5 x^{+3}+2 x^{-4}$ is a combination with Laurent polynomials generalizing decimal numbers: $3 x^{2}+4 x^{1}+5 x^{0}+6 x^{-1}+\left.7 x^{-2}\right|_{x-10}=345.67$.

We end with "the four operations" for Laurent polynomials. When adding and subtracting, the controlling fact is that $x^{ \pm n}$ behaves just like a denominator. Multiplication is easily dealt with by reading the code and, eventually, by using $\oplus$. After that we make a small detour to get $\left(x_{0}+h\right)^{n}=$ $x_{0}^{n}+n x_{0}^{n-1} h+\frac{n(n-1)}{2} x_{0}^{n-2} h^{2}+[\ldots]$ (usually not much further). The most important operation for us will be division, using $[\ldots]$ to stop it, as in $\frac{-12 x^{3}+11 x^{2}-17 x+1}{-3 x^{2}+5 x-2}=+4 x+3+8 x^{-1}+[\ldots]$

${ }^{4}$ Initially, students have a terrible time with even double basic problems. 


\section{Department of Mathematics}

\section{Research Assistant Professor}

(Ref. 1213/104(576)/2) (Closing date: May 15, 2013)

Applications are invited for a Research Assistant Professorship in any area of mathematics. Applicants should have a relevant $\mathrm{PhD}$ degree and good potential for research and teaching. Appointment will initially be made on contract basis for up to two years commencing August 2013, renewable subject to mutual agreement.

\section{Salary and Fringe Benefits}

Salary will be highly competitive, commensurate with qualifications and experience. The University offers a comprehensive fringe benefit package, including medical care, a contract-end gratuity for an appointment of two years, and housing benefits for eligible appointee. Further information about the University and the general terms of service for appointments is available at http://www.per.cuhk.edu.hk The terms mentioned herein are for reference only and are subject to revision by the University.

\section{Application Procedure}

Please send full resume, copies of academic credentials, a publication list and/or abstracts of selected published papers, together with names, addresses and fax numbers/e-mail addresses of three referees to whom the applicants' consent has been given for their providing references (unless otherwise specified), to the Personnel Office, The Chinese University of Hong Kong, Shatin, N.T., Hong Kong (Fax: (852) 3943 1462) by the closing date. The Personal Information Collection Statement will be provided upon request. Please quote the reference number and mark 'Application - Confidential' on cover. and

$$
\frac{-12+23 h-h^{2}-2 h^{3}}{-3+2 h}=+4-5 h-3 h^{2}+[\ldots] .
$$

\section{Conclusion}

I have reasons to believe that the contents sketched above: (i) can be absorbed by developmental students in a 5-hour course and (ii) can prepare developmental students for a two-semester (4-4) integrated alternative to the conventional (3-3-4) Precalculus I-Precalculus 2-Calculus 1 sequence that I developed under a 1988 NSF calculus grant:

- I am currently using part of the materials I developed for arithmetic along with the materials on inequations and on Laurent polynomial functions in a 3-hour developmental algebra course. The arithmetic materials which lead up to it should be doable with an additional 2 hours.

- The NSF sequence was based on Lagrange's approach, in which the $n$th derivative of $f$ at $x_{0}$ is defined (modulo $n$ !) as the coefficient of $x^{n}$ in the polynomial approximation of $f\left(x_{0}+h\right)$. As such, it does not require anything more than an Arithmetic-Algebra course along the lines sketched above.

So, given that

- The Office of Institutional Research at my institution found that "Of those attempting the first course in each sequence, 12.5\% finished the [conventional three-semester, 10-hour] sequence while $48.3 \%$ finished the [integrated two-semester, 8-hour] sequence." 5

- Having passed the Arithmetic-Algebra course and thus having already learned to take advantage of "mathematical compression", as well as having acquired precisely the necessary prerequisites, these formerly "unprepared students" ought now to hit the ground running in the first semester of the integrated alternative sequence and thus perform there at least as well as the students who entered the NSF sequence.

it would seem that, in contrast with the number given at the outset, 0.24 percent, such a three-semester (5-4-4) sequence should work for anywhere from about 5 percent to 20 percent of incoming "unprepared students". 6

\footnotetext{
${ }^{5}$ The passing rates in Calculus 2 were the same, but this was not really significant because of the low numbers of the students involved.

${ }^{6}$ The percentage would essentially depend on the extent to which the institution's placement test would be able to ascertain, instead of the students' "level of preparation", which is demonstrably meaningless in any case, their likely degree of commitment.
} 